\title{
Compromiso social como medida de reserva cognitiva y su relación con distintos procesos cognitivos en jóvenes universitarios
}

\author{
Eva Gabriela Trejo Becerra; Vicenta Reynoso-Alcántara
}

How to cite this article:

Trejo, E.G., \& Reynoso-Alcántara, V. (2019). Social engagement as a measurement of cognitive reserve and how it relates to different cognitive skills in college students. Acta Colombiana de Psicología, 22(2), $218-229$. doi: http://www.doi.org/10.14718/ACP.2019.22.2.11

Recibido, mayo 3/2018; Concepto de evaluación, noviembre 2/2018; Aceptado, enero 23/2019

\author{
Eva Gabriela Trejo Becerra \\ Universidad Veracruzana, Xalapa, México \\ ORCID: https://orcid.org/0000-0003-3895-3424 \\ Vicenta Reynoso-Alcántara* \\ Universidad Veracruzana, Xalapa, México \\ ORCID: https://orcid.org/0000-0003-1834-6117
}

\begin{abstract}
Resumen
La literatura científica indica que un mayor compromiso social se relaciona con un procesamiento cognitivo más eficiente, debido a que dicho compromiso incrementa la reserva cognitiva. Teniendo esto en cuenta, el objetivo de la presente investigación fue identificar la relación existente entre el compromiso social (en sus dimensiones de contacto, apoyo y conflicto social) y algunos procesos cognitivos (como flexibilidad mental, planeación, fluidez verbal, velocidad de procesamiento y comprensión verbal) en jóvenes universitarios. Para esto, se realizó un estudio correlacional con un diseño transversal en una muestra de 49 estudiantes. El análisis de correlación de Spearman reveló correlaciones entre contacto social y fluidez verbal $(r=-.299, p=.037)$, apoyo social y planeación $(r=.368, p=.009)$, y conflicto social y velocidad de procesamiento $(\mathrm{r}=.306, \mathrm{p}=.032)$. Estos resultados perfilan una asociación entre el compromiso social y algunas funciones cognitivas. A pesar de que los resultados no son del todo concluyentes, queda claro que se necesita de un estudio más detallado de estos procesos.

Palabras clave: reserva cognitiva, relaciones interpersonales, cognición.
\end{abstract}

\section{Social engagement as a measurement of cognitive reserve and how it relates to different cognitive skills in college students}

\begin{abstract}
Greater social engagement is commonly associated with more efficient cognitive processing, as it improves cognitive reserve. The aim of this research is to assess the relationship between social engagement (in the dimensions of social contact, support and conflict) and certain cognitive skills (cognitive flexibility, planning, verbal fluency, processing speed, and verbal comprehension) in young college students. This cross-sectional rank correlation study was carried out in a sample of 49 college students. Spearman's rank correlation coefficient showed relationship between: social contact and verbal fluency $(\mathrm{r}=-.299, \mathrm{p}=.037)$; social support and planning $(\mathrm{r}=.368, \mathrm{p}=.009)$; and social conflict and processing speed $(\mathrm{r}=.306$, $\mathrm{p}=.032$ ). The results outline a measurable relationship between social engagement and certain cognitive skills, even when said results are not entirely conclusive. Thus, the need for more detailed studies on these processes becomes clear.

Key words: cognitive reserve, interpersonal relationships, cognition.
\end{abstract}

Facultad de Psicología, Universidad Veracruzana. Manantial de San Cristóbal S/N. Xalapa 2000, CP 91097. Xalapa, Veracruz, México. Tel.: +52 2281268021. vreynoso@uv.mx 


\title{
Compromisso social como medida de reserva cognitiva e sua relaçáo com diferentes processos cognitivos em jovens universitários
}

\author{
Resumo
}

\begin{abstract}
A literatura científica indica que um maior compromisso social está relacionado com um pensamento cognitivo mais eficiente, devido ao fato de que tal compromisso aumenta a reserva cognitiva. Levando isso em consideração, o objetivo da presente pesquisa foi identificar a relação existente entre o compromisso social (em suas dimensões de contato, apoio e conflito social) e alguns processos cognitivos (como flexibilidade mental, planejamento, fluidez verbal, velocidade de processamento e compreensão verbal) em jovens universitários. Para tanto, realizou-se um estudo correlacional com um desenho transversal em uma amostra de 49 estudantes. A análise de correlação de Spearman revelou correlações entre contato social e fluidez verbal $(\mathrm{r}=-.299, \mathrm{p}=.037)$, apoio social e planejamento $(\mathrm{r}=.368, \mathrm{p}=.009)$ e conflito social e velocidade de processamento $(\mathrm{r}=.306$, $\mathrm{p}=.032$ ). Esses resultados constituem uma associação entre o compromisso social e algumas funções cognitivas. Apesar dos resultados não serem completamente conclusivos, fica clara a necessidade de um estudo mais detalhado desses processos.

Palavras-chave: reserva cognitiva, relações interpessoais, cognição
\end{abstract}

\section{Introducción}

En la actualidad, con el fin de mejorar la calidad de vida de las personas en general se busca extender el conocimiento en torno a los factores protectores que puedan favorecer el desarrollo de comunidades sanas en diversos ámbitos. En este sentido, dentro del ámbito de la salud mental se busca tanto incrementar el cuerpo de conocimiento sobre los factores que protegen a las personas de padecer deterioros cognitivos relacionados con el envejecimiento normal o patológico y con la presencia de diversas patologías que afectan la función cognitiva; como aumentar el conocimiento sobre los factores que promueven un estado cognitivo sano desde edades tempranas. Dentro de estos factores se incluyen, por ejemplo, los años de educación, el logro educativo, la complejidad laboral, el entorno enriquecido, la realización de actividades de esparcimiento y el compromiso social, entre otros (Lojo-Seoane, Facal, Juncos-Rabadán \& Pereiro, 2014; Mayor, Amador \& Ramírez, 2008; Seeman et al., 2011; Stern, 2009; Zhou, Wang \& Fang, 2018).

El interés en el estudio de estos temas deriva del proceso de transición demográfica orientado hacia el envejecimiento poblacional, con el cual se ha visto en aumento la prevalencia de enfermedades comunes en la vejez -como ocurre con la demencia-. Sin el desarrollo de las políticas necesarias para la prevención, las tendencias actuales hacia la longevidad podrían implicar un gran reto, ya que una población de adultos mayores enfermos requerirá de recursos de seguridad social y atenciones especiales que implican altos gastos que pueden conducir a una eventual crisis socioeconómica global (Lojo-Seoane et al., 2014; Mayor et al., 2008) y a que se vea deteriorada la calidad de vida de miles de personas.

Para contrarrestar y prevenir este problema, en el modelo de reserva cognitiva se propone que mediante una serie de actividades cognitivamente estimulantes desarrolladas a lo largo de la vida es posible generar una capacidad de reserva que parece tener un efecto protector u optimizador sobre la ejecución de los procesos cognitivos en todas las etapas de la vida, pero con especial relevancia durante el envejecimiento (Stern, 2009). Específicamente, la reserva cognitiva es definida por Stern (2009) como la habilidad para optimizar la ejecución mediante el reclutamiento diferencial de redes cerebrales que quizá reflejen el uso de estrategias cognitivas alternativas.

Al respecto, algunas evidencias apuntan a que elementos de riesgo y protección podrían ser acumulables a lo largo de la vida, por lo que tendrían implicaciones en la función cognitiva durante el envejecimiento; esto es lo que se conoce como reserva cognitiva (Nithianantharajah \& Hannan, 2009). Sin embargo, Stern (2009) propone que dicha reserva también puede tener un peso importante durante la juventud, aunque son escasos los trabajos realizados en población joven en los que se busque comprender cómo se construye desde edades tempranas la capacidad de hacer frente al deterioro cognitivo.

De igual forma, no existe un consenso sobre cuáles son las variables o indicadores que favorecen la formación de la reserva cognitiva, aunque frecuentemente se mencionan: (a) indicadores como la educación, la alfabetización, el bilingüismo y la capacidad intelectual general; (b) indicadores sociodemográficos como el estatus socioeconómico, la ocupación laboral y la ocupación de los padres; (c) indicadores directamente relacionados con la salud, como la nutrición y la realización de actividades físicas; (d) la realización de actividades cognitivamente estimulantes, dentro de las cuales se encuentran escribir, escuchar música, pintar, tener hábitos de lectura y participar en actividades culturales; y (e) actividades relacionadas con factores sociales, como realizar algún voluntariado y tener una vida socialmente activa (Lojo-Seoane et al., 2014; Manly et al., 2003; Mayor et al., 2008; Scarmeas \& Stern, 2003; Soto-Añari, FloresValdivia \& Fernández-Guinea, 2013). 
En particular, las relaciones sociales son uno de los indicadores de reserva cognitiva menos analizados en la literatura, a pesar de que, considerando su relevancia, podrían tener un papel preponderante para la formación y el mantenimiento de esta a lo largo de la vida. Dentro de lo que se encuentra en la literatura, algunas investigaciones exploran la asociación entre diferentes facetas de las relaciones sociales, tales como su tipo, diversidad o contenido, así como el compromiso social que las caracteriza, la autopercepción del contenido afectivo de las relaciones interpersonales (Seeman et al., 2011), y los efectos que producen sobre diversos procesos cognitivos y en la salud mental en general.

$\mathrm{Al}$ respecto, un amplio cuerpo de investigaciones ha demostrado la importancia de las relaciones sociales para la salud física y mental (Gardner, 2014; Gariépy, Honkaniemi \& Quesnel-Vallée, 2016; Zainab \& Naz, 2017; Zuelsdorff et al., 2018); al igual que asociaciones entre esta variable y lo que se define como un efecto protector contra el deterioro cognitivo, o la optimización de funciones cognitivas, principalmente en adultos mayores (Barnes, Mendes de Leon, Wilson, Bienias \& Evans, 2004; Bassuk, Glass \& Berkman, 1999; Bennett, Schneider, Tang, Arnold \& Wilson, 2006; Berkman, 2000; Fratiglioni, Paillard-Borg \& Winblad, 2004; Krueger \& Wilson, 2009; Marioni et al., 2015; Nelson, Noonan, Goldberg \& Buchwald, 2013; Pillemer \& Holtzer, 2016; Rodriguez-Laso, Zunzunegui \& Otero, 2007; Saczynski, 2006; Seeman et al., 2011; Seeman, Lusignolo, Albert \& Berkman, 2001; Tun, Miller-Martinez, Lachman \& Seeman, 2013; Wilson et al., 2007; Zhou et al., 2018; Zunzunegui, Alvarado, Del Ser \& Otero, 2003).

En particular, por relación social se entiende un compromiso recíproco basado en interacciones que dan lugar a tipos específicos de confianza entre los individuos (Grossetti, 2009), y en este sentido, el efecto que las relaciones sociales pueden llegar a tener sobre el cerebro humano y las funciones cognitivas es innegable, ya que los seres humanos son criaturas sociales con especial habilidad para los procesos cognitivos y de comunicación de orden superior, los cuales podrían haber evolucionado, en parte, por la necesidad de llevar un seguimiento de las relaciones sociales y de guiar el comportamiento social complejo (Adolphs, 2003).

Por otra parte, se ha encontrado que las experiencias sociales tienen un impacto sobre las respuestas fisiológicas del cerebro, por lo que se propone que el efecto protector de la participación en actividades sociales sobre el deterioro cognitivo puede darse debido a que dichas relaciones fomentan la comunicación y las interacciones interpersonales, y, por tanto, cierta estimulación cognitiva que puede ayudar en la reducción del deterioro cognitivo (Berkman,
2000). Es importante señalar que se ha encontrado que los efectos de las relaciones sociales dependen de la naturaleza de estas, es decir, que mientras el apoyo social implicaría beneficios, el conflicto social y la tensión con las personas de las redes sociales implicarían consecuencias negativas (Gariépy et al., 2016; Kiecolt-Glaser \& Newton, 2001; Liao et al., 2014).

Más allá de esto, Seeman et al. $(2001,2011)$ proponen un constructo multidimensional denominado compromiso social para referirse a los aspectos cuantitativos y cualitativos de la interacción social; y en la Encuesta nacional del desarrollo de la mediana edad (MIDUS I; 1995/1996) en los EE. UU., y en su segunda oleada (MIDUS II; 2005/2006), dichos autores evaluaron tres dimensiones del compromiso social -el contacto social, el apoyo social y el conflicto social y su relación con la función ejecutiva-, donde encontraron que las historias de mayor frecuencia de contactos sociales e interacciones de apoyo se asociaron positivamente con funciones ejecutivas, mientras que la tensión o conflicto y la disminución de los contactos sociales correlacionaron negativamente con ellas (Cornwell \& Laumann, 2015; Seeman et al., 2011; Segel-Karpas \& Lachman, 2016; Tun et al., 2013).

Asimismo, se ha encontrado que el apoyo social percibido predice una mejor función cognitiva (Pillemer \& Holtzer, 2016; Seeman et al., 2001), mientras que las percepciones sociales negativas se asocian con bajos desempeños en una amplia gama de funciones cognitivas, incluidas la velocidad de procesamiento y la función ejecutiva (Tun et al., 2013). De igual forma, se ha encontrado que el tamaño de las redes sociales correlaciona con la salud mental en general, ya que redes grandes se vinculan con mayores niveles de salud mental (Rodriguez-Laso et al., 2007; Umberson \& Karas, 2010), y que, incluso, modifican la asociación entre la patología y la función cognitiva (Bennett et al., 2006).

En la investigación gerontológica, además, se ha encontrado que las diferentes configuraciones de las redes sociales y el apoyo social influyen en el estado neuropsicológico, ya que, por ejemplo, la participación en redes sociales de congregación o diversidad se ha asociado significativamente con buena salud mental y menor probabilidad de padecer deterioro cognitivo (Marioni et al., 2015).

Del mismo modo, hay evidencia de que el nivel de compromiso social en adultos mayores puede predecir el riesgo de presentar deterioro cognitivo u otros padecimientos (Barnes et al., 2004; Bassuk et al., 1999; Holtzman et al., 2004; Krueger \& Wilson, 2009; Liao \& Scholes, 2017; Wilson et al., 2015; Zunzunegui et al., 2003), al igual que el riesgo de padecer demencia (Bennett, Arnold, Valenzuela, Brayne \& Schneider, 2014; Marioni et al., 2015; Saczynski, 
2006; Skakkebæk et al., 2017; Zhou et al., 2018). Seeman et al. (2011) plantean la posibilidad de que estas asociaciones -positivas o negativas- entre el apoyo u otros aspectos de la interacción social y los procesos cognitivos podrían ser evidentes también en la juventud y no solo en adultos mayores.

Es importante señalar que la correlación entre el compromiso social y una mejor función cognitiva varía a través de los distintos dominios o subcomponentes del compromiso social y los diferentes procesos cognitivos (Nelson et al., 2013). Específicamente, los estudios en los que se analiza la relación entre el compromiso social y las funciones cognitivas incluyen diversas funciones, ya que, por ejemplo, la memoria, el razonamiento, la fluidez verbal, el vocabulario, la visualización espacial y la velocidad de procesamiento se relacionan positivamente con el contacto y el apoyo social (La Fleur \& Salthouse, 2017; Seeman et al., 2011); mientras que la velocidad de procesamiento se relaciona negativamente con el conflicto social (Tun et al., 2013); y un índice global de función cognitiva -que incluyó memoria, atención, lenguaje y habilidades visoespaciales-se relacionó positivamente con la interacción social positiva y con el apoyo emocional percibido (Pillemer \& Holtzer, 2016). Del mismo modo, un índice global de función cognitiva -que consideró memoria episódica, memoria semántica, velocidad perceptual y habilidad visoespacial-se relacionó positivamente con el tamaño de las redes sociales (Wilson et al., 2007).

Teniendo en cuenta la importancia de las funciones ejecutivas para que los seres humanos alcancen objetivos complejos, especialmente cuando estos requieren de un abordaje novedoso (Lezak, Howieson, Bigler \& Tranel, 2012), resulta relevante comprender con mayor detalle la relación entre dichas funciones y el compromiso social -considerado como una medida de reserva cognitiva-.

Ahora bien, las funciones ejecutivas pueden definirse como un conjunto de habilidades y capacidades implicadas en generar, planear, controlar, regular, ejecutar y reajustar las conductas y los procesos cognitivos necesarios para alcanzar objetivos complejos (Lezak et al., 2012); las cuales comienzan a desarrollarse durante el primer año de vida y lo continúan haciendo a lo largo del ciclo vital, de modo que, incluso en la vida adulta, pueden agilizarse y mejorarse mediante la estimulación adecuada (Rosselli, Jurado \& Matute, 2008).

Dentro de las funciones ejecutivas, se incluyen, entre otras, la flexibilidad mental, que es la capacidad de inhibir una respuesta o estrategia cognitiva equivocada, evitar la tendencia a utilizarla repetitivamente y generar una respuesta alternativa apropiada para una tarea específica; la planeación secuencial, entendida como la habilidad para ejecutar ordenadamente una serie de pasos que en conjunto conducen a una meta específica; y la fluidez verbal, que refleja la medida de la eficiencia para producir el mayor número posible de palabras en un tiempo límite determinado a partir de un criterio específico (Flores, Ostrosky \& Lozano, 2014).

Finalmente, algunos otros procesos cognitivos que han sido estudiados en relación con la reserva cognitiva son la velocidad de procesamiento y la comprensión verbal, para los cuales se puede utilizar la definición operacional de Wechsler (2012) para los índices del WAIS-IV, en la que el indice de velocidad de procesamiento es la medida de la capacidad de la persona para explorar, secuenciar o discriminar información visual simple de forma rápida y correcta; mientras que el índice de comprensión verbal es una medida de los procesos de formación de conceptos y el razonamiento verbal, así como del conocimiento adquirido del ambiente.

Teniendo en cuenta los antecedentes mencionados, el objetivo del presente trabajo fue identificar las correlaciones existentes entre el compromiso social, las funciones ejecutivas -flexibilidad mental, planeación secuencial y fluidez verbal-, la velocidad de procesamiento y la comprensión verbal en adultos jóvenes mexicanos. Haciendo una extrapolación de los hallazgos con adultos mayores en estudios previos a la población de jóvenes, se propuso como hipótesis que un mayor contacto y apoyo social se relacionarán con mayores puntuaciones en las funciones ejecutivas, la velocidad de procesamiento y la comprensión verbal; y que un mayor conflicto social se asociará con menores puntuaciones en las funciones ejecutivas, la velocidad de procesamiento y la comprensión verbal.

\section{Método}

Se realizó un estudio de corte transversal, correlacional (Hernández, Fernández \& Baptista, 2010), con un protocolo de investigación aprobado por el Comité de Ética (No. de registro CEI-PSI/006/2016) de la entidad donde se llevó a cabo la investigación. Todos los participantes firmaron un consentimiento informado.

\section{Participantes}

Se realizó un muestreo no probabilístico por conveniencia, a partir del cual se obtuvo una muestra conformada por 49 jóvenes estudiantes de una universidad pública con visión y audición normal o corregida a nivel normal. El 32.7 \% de los participantes eran mujeres y el $67.3 \%$, hombres, 
ambos con edades entre los 18 y los 27 años, y una media de edad de $20.82(\mathrm{DE}=2.01)$. Por medio de una entrevista estructurada que retoma algunos criterios que forman parte del cuestionario neuropsicológico de daño frontal, incluido en el BANFE-2 (Flores et al., 2014), se descartaron los jóvenes que cumplieran alguno de los siguientes criterios de exclusión: historial de perturbaciones neurológicas o psiquiátricas, desórdenes del desarrollo neurológico (deterioro intelectual, trastornos del aprendizaje, déficit de atención con hiperactividad, etc.), patologías físicas (trastornos del sueño, síndromes cardíacos, epilepsia y trauma en la cabeza) y uso de drogas ilegales. Finalmente, mediante el Test de identificación de trastornos por consumo de alcohol (AUDIT; Cremonte, Ledesma, Cherpitel \& Borges, 2010), se descartó a los estudiantes que indicaban abuso de alcohol. No se aplicó ninguna prueba para descartar alguna condición emocional desfavorable independiente de las ya mencionadas anteriormente.

\section{Instrumentos}

Se diseñó e implementó el Cuestionario de Compromiso Social, un instrumento que mide el compromiso social diferenciadamente en las esferas familia, amigos y pareja, a partir del cual se obtienen tres medidas: un indicador de contacto social, que refleja la frecuencia de interacciones interpersonales que sostiene el participante con los grupos de contacto; un indicador del apoyo social, que refleja las interacciones sociales armoniosas; y un indicador de conflicto social, que refleja las interacciones de tensión y de conflicto.

Para su elaboración, se tomaron como referencia las medidas utilizadas en el MIDUS I (1994/1995) y el MIDUS II (2005/2006). Debido a que no se encuentra validado para población mexicana, como parte de esta investigación se llevó a cabo una aplicación piloto del instrumento en una muestra de 27 estudiantes que contaban con características similares a las de la muestra utilizada en este estudio -estudiantes jóvenes de ambos sexos de la facultad de psicología en una universidad pública-, donde se obtuvo un alfa de Cronbach de .806. En la posterior aplicación desarrollada en esta investigación se obtuvo un alfa de Cronbach de.801.

Por otra parte, las funciones ejecutivas se evaluaron por medio de algunas subpruebas de la BANFE-2 (Flores et al., 2014), entre las que se incluyeron clasificación de cartas, para evaluar flexibilidad mental; torre de Hanói, para evaluar planeación secuencial; y la tarea de fluidez verbal, para evaluar la fluidez verbal. El BANFE-2 cuenta con validación para su uso en población mexicana. La confiabilidad reportada en el manual para estas pruebas es de .80 .
El resto de los procesos cognitivos se evaluaron con algunas subpruebas de la escala WAIS-IV (Wechsler, 2012). Específicamente, se utilizó la subprueba de búsqueda de símbolos (con confiabilidad de .81 reportada en el manual de la prueba) y de claves (con confiabilidad de .86 reportada en el manual de la prueba) para la medición de la velocidad de procesamiento. Finalmente, se utilizó la subprueba de vocabulario (con confiabilidad de .92 reportada en el manual de la prueba) para la medición de la comprensión verbal. El WAIS-IV cuenta con validación para su uso en población mexicana.

\section{Procedimiento}

Se realizó una convocatoria en un campus de una universidad pública mexicana para participar en la investigación. Los interesados leyeron y firmaron el consentimiento informado, $\mathrm{y}$ cada participante asistió a una sesión individual única llevada a cabo en un espacio apropiado para tal fin. Por medio de una entrevista, se verificó que los interesados cumplieran con los criterios de inclusión, y, posteriormente, se aplicó el cuestionario de compromiso social en modo de entrevista. Las pruebas utilizadas se aplicaron y calificaron de acuerdo con la forma estandarizada marcada en los manuales. Tanto la entrevista como las pruebas fueron realizadas por personal capacitado. Finalmente, se creó una base de datos en la que se codificaron las respuestas, y se utilizó el programa SPSS (SPSS 16.0 para Windows, SPSS Inc., Chicago, IL) para realizar los análisis pertinentes.

\section{Análisis de datos}

Se llevaron a cabo análisis estadísticos descriptivos para conocer las frecuencias, porcentajes, media, mediana y desviación estándar tanto de las variables evaluadas como de las características de la población, tales como edad y sexo. Posteriormente, atendiendo al objetivo de identificar las relaciones existentes entre el compromiso social y algunos procesos cognitivos en jóvenes, se realizaron correlaciones bivariadas. Se seleccionó el coeficiente de correlación de Spearman debido a que las variables no contaban con una distribución normal.

\section{Resultados}

En este apartado se presentan los resultados obtenidos en la investigación, iniciando por los resultados de estadística descriptiva de cada variable evaluada: compromiso social -en sus dimensiones de contactos sociales, apoyo social y tensión o conflicto social-, funciones ejecutivas 
-flexibilidad mental, capacidad de planeación secuencial y fluidez verbal- y procesos cognitivos -velocidad de procesamiento y comprensión verbal-. Posteriormente se presentan los resultados sobre el grado de correlación entre el compromiso social -en sus tres dimensiones- y las funciones ejecutivas, la velocidad de procesamiento y la comprensión verbal de los jóvenes, respectivamente.

\section{Compromiso social}

En la Tabla 1 se presentan los resultados descriptivos obtenidos a partir de los datos de compromiso social, considerando las dimensiones de contacto social, apoyo social y conflicto social en las categorías de familia, amigos, pareja y el total. Se debe señalar que en la variable apoyo social mayores puntuaciones implicaron menor apoyo social, y que en la variable conflicto social mayores puntuaciones implicaron menor conflicto. Esto debido a que se conservó el formato del cuestionario original, base de la versión modificada aplicada en este trabajo.

En general, se observa que el mayor contacto social de los participantes se da en la esfera de "familia", sin embargo, según lo reportado, es en esta esfera en la que manifiestan recibir menos apoyo social, mientras que el mayor apoyo se recibe de la esfera de "pareja". Con respecto a conflicto, la esfera de "amigos" es de la que se reporta como con menor conflicto, mientras que el mayor conflicto se reporta en la esfera de "pareja". Con respecto a la esfera de "pareja", cabe mencionar que únicamente el $36.73 \%$ de participantes manifestaron tener pareja, por lo que los resultados reportados podrían ser poco relevantes.

En la Tabla 1 se observa una menor dispersión en el contacto social que en el caso del apoyo social y el conflicto social; y que dentro de los dominios contacto social, apoyo social y conflicto social, la categoría "pareja" contó con puntuaciones menores, esto debido a que, de una muestra de 49 participantes, 18 no se encontraban dentro de una relación formal o informal. También, puede notarse que el dominio conflicto social tuvo puntuaciones más elevadas para la categoría "amigos", ya que puntuaciones más elevadas para este dominio representan menor conflicto social; esto indicó que en la muestra se presentó menor conflicto dentro de relaciones de amigos, en comparación con relaciones de familia y pareja.

\section{Procesos cognitivos}

Con intención descriptiva, y para su comparación con otras investigaciones, en la Tabla 2 se presentan las medias, desviación estándar y medianas de las medidas consideradas en el presente trabajo para los procesos cognitivos. Dentro de los datos presentados, se incluyen, específicamente, lo referente a flexibilidad mental, con las puntuaciones de la prueba de clasificación de cartas; planeación secuencial, con las puntuaciones de la prueba de torre de Hanói; fluidez verbal, con la prueba que lleva este mismo nombre; la velocidad de procesamiento, con las puntuaciones de las subpruebas búsqueda de símbolos y claves; y comprensión verbal, con las puntuaciones de la subprueba de vocabulario.

En general, se observa que en el caso de los criterios de clasificación de cartas - perseveraciones, clasificación de cartas - perseveraciones diferidas, clasificación de cartas - errores de mantenimiento, torre de Hanói - movimientos, torre de Hanói - error A, torre de Hanói - error B, torre de Hanói - errores totales y fluidez verbal - intrusiones, la desviación estándar fue mayor a la media, debido a la presencia de casos atípicos con puntuaciones con mayor distancia del promedio en puntuaciones altas o bajas (véase Tabla 2).

Correlaciones entre compromiso social y procesos cognitivos

En la Tabla 3 se presentan las correlaciones encontradas entre los procesos cognitivos evaluados y el contacto social, apoyo social y conflicto social.

Con respecto al contacto social, se encontró una correlación negativa débil, pero significativa $(\mathrm{r}=-.299, \mathrm{p}=.037)$, entre el contacto social en la esfera "amigos" y la medida de intrusiones de la tarea de fluidez verbal (mencionar

Tabla 1.

Estadistica descriptiva del compromiso social

\begin{tabular}{lcccccc}
\hline \multirow{2}{*}{ Esfera } & Contacto social & \multicolumn{3}{c}{ Apoyo social } & Conflicto social \\
\cline { 2 - 7 } & $\mathrm{M}(\mathrm{DE})$ & $\mathrm{Me}$ & $\mathrm{M}(\mathrm{DE})$ & $\mathrm{Me}$ & $\mathrm{M}(\mathrm{DE})$ & $\mathrm{Me}$ \\
\hline Familia & $3.59(0.762)$ & 4.00 & $6.71(2.092)$ & 7.00 & $10.88(2.360)$ & 11.00 \\
Amigos & $3.53(0.739)$ & 4.00 & $6.29(1.860)$ & 6.00 & $12.20(2.041)$ & 12.00 \\
Pareja & $2.39(1.891)$ & 4.00 & $3.63(3.053)$ & 4.00 & $7.96(6.409)$ & 11.00 \\
Total & $3.18(0.65)$ & 3.33 & $5.57(1.59)$ & 5.33 & $10.30(2.53)$ & 10.00 \\
\hline
\end{tabular}

Nota. Estadística descriptiva de los datos de compromiso social en sus dimensiones contacto social, apoyo social, conflicto social, en las categorías familia, amigos, pareja y el total. 
Tabla 2.

Estadística descriptiva de procesos cognitivos

\begin{tabular}{|c|c|c|}
\hline Medida & $\mathrm{M}(\mathrm{DE})$ & $\mathrm{Me}$ \\
\hline \multicolumn{3}{|c|}{ Funciones ejecutivas: Flexibilidad mental } \\
\hline Clasificación de cartas - Aciertos & $46.51(9.16)$ & 49.00 \\
\hline Clasificación de cartas - Errores & $10.94(3.96)$ & 10.00 \\
\hline Clasificación de cartas - Perseveraciones & $4.67(5.39)$ & 3.00 \\
\hline Clasificación de cartas - Perseveraciones diferidas & $1(1.36)$ & .00 \\
\hline Clasificación de cartas - Errores de mantenimiento & $0.47(0.819)$ & .00 \\
\hline Clasificación de cartas - Tiempo & $338.18^{\mathrm{a}}\left(112.08^{\mathrm{a}}\right)$ & 313.00 \\
\hline \multicolumn{3}{|c|}{ Funciones ejecutivas: Planeación secuencial } \\
\hline Torre de Hanói - Movimientos & $34.22(50.38)$ & 24.00 \\
\hline Torre de Hanói - Error A & $0.51(1.02)$ & .00 \\
\hline Torre de Hanói - Error B & $0.37(1.51)$ & .00 \\
\hline Torre de Hanói - Errores totales & $0.88(1.78)$ & .00 \\
\hline Torre de Hanói - Tiempo & $109^{\mathrm{a}}\left(63.09^{\mathrm{a}}\right)$ & 98.00 \\
\hline \multicolumn{3}{|c|}{ Funciones ejecutivas: Fluidez verbal } \\
\hline Fluidez verbal - Aciertos & $20.29(6.04)$ & 20.00 \\
\hline Fluidez verbal - Intrusiones & $0.08(0.27)$ & .00 \\
\hline Fluidez verbal - Perseveraciones & $0.63(0.95)$ & .00 \\
\hline \multicolumn{3}{|c|}{ Velocidad de procesamiento } \\
\hline Búsqueda de símbolos - Puntuación escalar & $10.24(1.877)$ & 10.00 \\
\hline Claves - Puntuación escalar & $10.69(1.960)$ & 10.00 \\
\hline \multicolumn{3}{|c|}{ Comprensión verbal } \\
\hline Vocabulario - Puntuación escalar & $7.41(1.48)$ & 7.00 \\
\hline
\end{tabular}

una palabra que no corresponde a la categoría indicada), donde a mayor contacto social en la esfera de "amigos", menos intrusiones.

Con respecto al apoyo social, se encontró una correlación positiva débil, pero significativa $(r=.368, \mathrm{p}=.009)$, entre el apoyo social en la esfera de "amigos" y la planeación secuencial en el error B de la torre de Hanói (colocar un disco de mayor tamaño sobre otro más pequeño, contrario a la instrucción), donde a mayor puntuación en apoyo social en la esfera de "amigos" - es decir, menor presencia de relaciones de apoyo-, más errores de tipo B en torre de Hanói.

Finalmente, al considerar el conflicto social, se encontró una correlación positiva débil, pero significativa $(\mathrm{r}=.306$, $\mathrm{p}=.032$ ), entre la puntuación total -donde se engloba en una sola medida las tres esferas de contacto: familia, amigos y pareja- y la subprueba búsqueda de símbolos, donde a mayor puntuación en conflicto social -es decir, menor presencia de relaciones de conflicto-, mejor ejecución en la subescala. Y, por último, se encontró una correlación positiva débil, pero significativa $(r=.302, p=.035)$, entre el conflicto social en la esfera de "familia" y la subprueba de búsqueda de símbolos, donde a menor presencia de relaciones de conflicto, mejor ejecución en la subescala.

\section{Discusión}

Los resultados presentados dan cuenta de correlaciones significativas entre el compromiso social y algunas medidas de función cognitiva en el grupo de jóvenes participantes; esto teniendo en cuenta el contacto, el apoyo y el conflicto social, sobre todo en las esferas de "amigos" y "familia". 
Trejo Becerra, E.G. \& Reynoso-Alcántara, V.

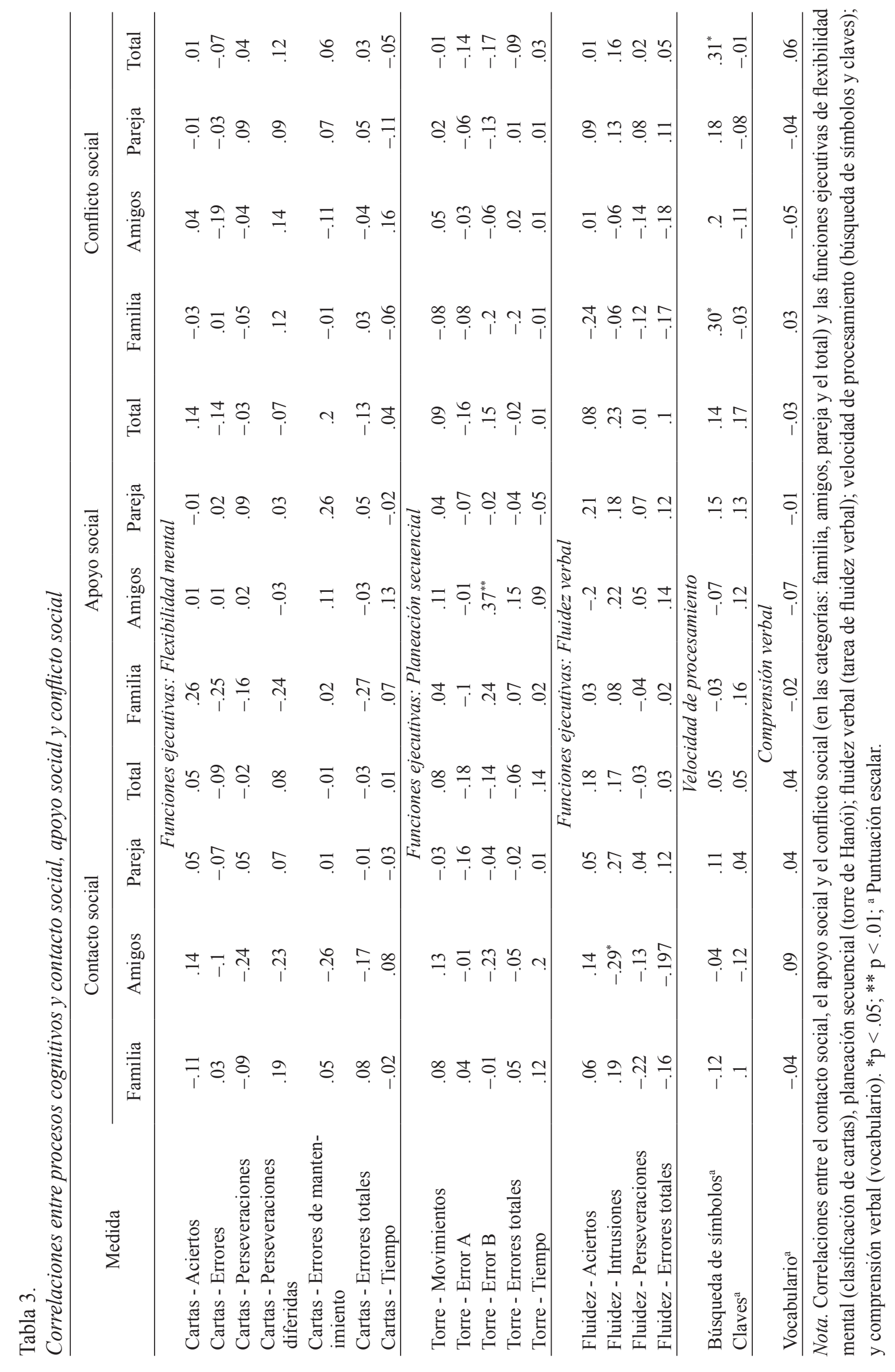


Sin embargo, no se observaron correlaciones en todas las medidas de función cognitiva evaluadas. Específicamente, las únicas medidas de función cognitiva que tienen relación con el compromiso social fueron las intrusiones en fluidez verbal, el error B de la torre de Hanói -en planeación secuencial- y la puntuación escalar de la subprueba búsqueda de símbolos en la evaluación de la velocidad de procesamiento. Las correlaciones observadas son, en todos los casos, débiles.

Según la literatura, se esperaba que, entre mayor contacto social y apoyo social, mejor ejecución en las funciones cognitivas evaluadas, y en el presente trabajo se encontró que una mayor frecuencia de contacto social en la esfera "amigos" se asocia con menos errores de intrusión en fluidez verbal. Por tanto, es probable que la asociación entre la fluidez verbal y frecuencia del contacto social refleje que el contacto social constante implica la participación en actividades que involucran la utilización de recursos y capacidades de comunicación que ejercitan la capacidad comunicativa, de modo que esta se vuelve más eficiente, tal como lo postulan Berkman (2000) y McEwen (2007).

Asimismo, se encontró que menor presencia de apoyo social en la esfera de "amigos" se relaciona con más errores en planeación secuencial. Esto podría explicarse bajo la premisa de que la historia acumulativa de un individuo y sus interacciones sociales, o la falta de las mismas, tienen consecuencias sobre la adaptación del cerebro y los sistemas cognitivos que intervienen en la forma de percibir, interpretar y recordar (Tun et al., 2013).

Por otro lado, se esperaba que una mayor presencia de conflicto social se relacionara con una peor ejecución en las funciones cognitivas. En este sentido, los resultados dieron cuenta de que, a menor presencia de relaciones de conflicto, mejor ejecución en la subescala de búsqueda de símbolos; lo cual supone una posible expresión del efecto favorable de la ausencia de conflicto social sobre la velocidad de procesamiento. Estos resultados son consistentes con el trabajo de Tun et al. (2013), en el cual se encontró una asociación entre las variables de conflicto social y velocidad de procesamiento, por lo que se podría suponer que la velocidad de procesamiento es especialmente vulnerable a los efectos de la tensión social no solo en adultos mayores, sino también jóvenes. Los resultados también fueron parcialmente consistentes con el trabajo de Seeman et al. (2011), ya que ambos estudios coinciden en poner en evidencia la relación del compromiso social -el conflicto social, en especial- con la cognición antes de la vejez.

Aunque las evidencias presentadas sobre la existencia de una relación entre el compromiso social y los procesos cognitivos en jóvenes podrían resultar poco concluyentes, estos resultados contrastan con lo reportado en adultos mayores, en los que se reporta que el compromiso social es una variable que se asocia de forma importante con el desempeño cognitivo (Barnes et al., 2004; Bassuk et al., 1999; Holtzman et al., 2004; Krueger \& Wilson, 2009; Zhou et al., 2018; Zunzunegui et al., 2003); por lo tanto, pareciera que el compromiso social podría no ser tan relevante para la eficiencia cognitiva en jóvenes, aun cuando hay indicios de que relaciones interpersonales menos conflictivas se asocian con una mayor velocidad de procesamiento. Teniendo esto en cuenta, a pesar de que en la presente investigación se encontró una relación, en general, débil entre el compromiso social y los procesos cognitivos, esta área requiere de más investigación antes de negar la posible asociación de tales variables y su expresión en la juventud.

Ahora bien, es importante mencionar algunos factores que pudieron condicionar los resultados e, incluso, influir en que no se encontraran las correlaciones propuestas en las hipótesis.

Con respecto al diseño de investigación, no se realizaron comparaciones entre grupos, pero el posible efecto optimizador del compromiso social podría evidenciarse con mayor claridad al comparar grupos numerosos con alta y baja reserva cognitiva, o grupos de adultos jóvenes y adultos mayores, ante tareas de demanda incrementada. Este tipo de tareas habría permitido comparar el desempeño de los participantes para apreciar el posible efecto optimizador de la reserva cognitiva (Mayor et al., 2008; Stern, 2009). También, en cuanto al diseño, al tratarse de una investigación trasversal, no es posible corroborar el efecto acumulativo de la reserva cognitiva evaluada a partir del compromiso social.

Con respecto a las limitaciones de los instrumentos aplicados, no se aplicó ninguna prueba para descartar alguna condición emocional desfavorable como parte de los criterios de exclusión; además de que, en cuanto a las mediciones de los procesos cognitivos, se utilizaron como referencia del desempeño en las funciones ejecutivas puntuaciones crudas de algunas de las subpruebas que conforman la BANFE-2 (Flores et al., 2014), sin embargo, esta batería no proporciona índices individuales o puntuaciones escalares para sus subpruebas -solo índices globales de funcionamiento-, ni proporciona información más específica, como los tiempos de reacción, que son más sensibles a las diferencias entre grupos.

En cuanto a las mediciones del compromiso social, el cuestionario aplicado evalúa un solo momento del ciclo de vida, no la historia de interacciones sociales a lo largo de este; aspecto que reflejaría mejor el efecto de la reserva cognitiva, puesto que esta es acumulable. También, dentro de la esfera "pareja", únicamente el $36.73 \%$ de los participantes manifestó tener una relación de este tipo, por lo 
que los resultados reportados podrían no ser relevantes. También, cabe considerar las limitaciones de este cuestionario con respecto a la normalización de los resultados y su interpretación, puesto que solo se probó el instrumento en una prueba piloto. Finalmente, es pertinente recordar que el compromiso social es un constructo multidimensional, y que la manera en que sea definido conceptual y operacionalmente se relaciona directamente con los resultados obtenidos.

También, en lo concerniente a la muestra evaluada, esta estuvo conformada por una cantidad pequeña de participantes y fue obtenida a partir de un muestreo no probabilístico por conveniencia. Además, todos los participantes coincidían con un mínimo común de formación académica, lo que pudo homogenizar su desempeño en las subpruebas aplicadas, ya que la educación medida en años de educación formal es la variable de la cual existe mayor evidencia de su correlación con la optimización de procesos cognitivos (Mayor et al., 2008).

Por último, al tratarse de una muestra compuesta únicamente por estudiantes universitarios, los resultados carecen de la representatividad necesaria para ser generalizados a todo tipo de jóvenes de manera independiente a su formación académica; además, es posible que en la etapa del desarrollo en que se encontraban los participantes no se aprecien los efectos de la reserva cognitiva, o al menos no en relación con los procesos cognitivos evaluados.

Para llegar a resultados generalizables que contribuyan a precisar el tipo de estimulación que favorece la optimización de los procesos cognitivos en poblaciones jóvenes y sanas, investigaciones futuras requerirán analizar la relación entre variables sociales -como el compromiso social en sus distintas dimensiones- $-\mathrm{y}$ distintos procesos cognitivos en diferentes contextos, poblaciones y momentos del ciclo de vida. Esta investigación es un punto de partida en el que se subraya la relevancia del estudio de estos tópicos en relación con la salud mental.

\section{Referencias}

Adolphs, R. (2003). Cognitive neuroscience: Cognitive neuroscience of human social behaviour. Nature Reviews Neuroscience, 4(3), 165-178. doi: 10.1038/nrn1056

Barnes, L. L., Mendes de Leon, C. F., Wilson, R. S., Bienias, J. L., \& Evans, D. A. (2004). Social resources and cognitive decline in a population of older African Americans and whites. Neurology, 63(12), 2322-2326. doi: 10.1212/01. WNL.0000147473.04043.B3

Bassuk, S. S., Glass, T. A., \& Berkman, L. F. (1999). Social Disengagement and Incident Cognitive
Decline in Community-Dwelling Elderly Persons. Annals of Internal Medicine, 131(3), 165-173. doi: 10.7326/0003-4819-131-3-199908030-00002

Bennett, D. A., Arnold, S. E., Valenzuela, M. J., Brayne, C., \& Schneider, J. A. (2014). Cognitive and social lifestyle: Links with neuropathology and cognition in late life. Acta Neuropathological, 127(1), 137-150. doi: 10.1007/ s00401-013-1226-2

Bennett, D. A., Schneider, J. A., Tang, Y., Arnold, S. E., \& Wilson, R. S. (2006). The effect of social networks on the relation between Alzheimer's disease pathology and level of cognitive function in old people: a longitudinal cohort study. Lancet Neurology, 5(5), 406-412. doi: 10.1016/ S1474-4422(06)70417-3

Berkman, L. F. (2000). Which influences cognitive function: Living alone or being alone? The Lancet, 355(9212), 12911292. doi: 10.1016/S0140-6736(00)02107-3

Cornwell, B., \& Laumann, E. O. (2015). The health benefits of network growth: New evidence from a national survey of older adults. Social Science Medicine, 125, 94-106. doi: 10.1016/j.socscimed.2013.09.011

Cremonte, M., Ledesma, R., Cherpitel, C., \& Borges, G. (2010). Psychometric properties of alcohol screening tests in the emergency department in Argentina, Mexico and the United States. Addictive Behaviors, 35(9), 818-25. doi: 10.1016/j. addbeh.2010.03.021

Flores, J. C., Ostrosky, F., \& Lozano, A. (2014). BANFE-2, Batería neurosicológica de funciones ejecutivas y lóbulos frontales. México: Manual Moderno.

Fratiglioni, L., Paillard-Borg, S., \& Winblad, B. (2004). An active and socially integrated lifestyle in late life might protect against dementia. Lancet Neurology, 3(6), 343-353. doi: 10.1016/S1474-4422(04)00767-7

Gardner, P. (2014). The role of social engagement and identity in community mobility among older adults aging in place. Disability and Rehabilitation, 36(15), 1249-1257. doi:10.3 109/09638288.2013.837970

Gariépy, G., Honkaniemi, H., \& Quesnel-Vallée, A. (2016). Social support and protection from depression: systematic review of current findings in Western countries. British Journal of Psychiatry, 209(4), 284-293. doi: 10.1192/bjp. bp.115.169094

Grossetti, M. (2009). ¿Qué es una relación social? Un conjunto de mediaciones diádicas. REDES-Revista Hispana Para El Análisis de Redes Sociales, 6(2), 44-62. doi: 10.5565/REV/ REDES.364

Hernández, R., Fernández, C., \& Baptista, P. (2010). Metodología de la investigación (5. a ed.). México: McgrawHill Educación.

Holtzman, R. E., Rebok, G. W., Saczynski, J. S., Kouzis, A. C., Wilcox Doyle, K., \& Eaton, W. W. (2004). Social network characteristics and cognition in middle-aged and older adults. The Journals of Gerontology: Psychological Sciences, 59(6), 278-284. doi: 10.1093/geronb/59.6.P278 
Kiecolt-Glaser, J. K., \& Newton, T. L. (2001). Marriage and health: His and hers. Psychological Bulletin, 127(4), 472503. doi: 10.1037//0033-2909.127.4.472

Krueger, K., \& Wilson, R. (2009). Social engagement and cognitive function in old age. Experimental Aging Research, 35(1), 1-12. doi: 10.1080/03610730802545028

La Fleur, C. G., \& Salthouse, T. A. (2017). Which aspects of social support are associated with which cognitive abilities for which people? The journals of gerontology. Series B, Psychological sciences and social sciences, 72(6), 10061016. doi: 10.1093/geronb/gbv119

Lezak, M., Howieson, D., Bigler, E., \& Tranel, D. (2012). Neuropsychological Assessment (5. ${ }^{\text {a }}$ ed.). New York: Oxford University Press.

Liao, J., \& Scholes, S. (2017). Association of social support and cognitive aging modified by sex and relationship type: A prospective investigation in the English longitudinal study of ageing. American Journal of Epidemiology, 186(7), 787795. doi: 10.1093/aje/kwx142

Liao, J., Head, J., Kumari, M., Stansfeld, S., Kivimaki, M., Singh-Manoux, A., \& Brunner, E. J. (2014). Negative aspects of close relationships as risk factors for cognitive aging. American Journal of Epidemi, 180(11), 1118-1125. Doi: 10.1093/aje/kwu236

Lojo-Seoane, C., Facal, D., Juncos-Rabadán, O., \& Pereiro, A. X. (2014). El nivel de vocabulario como indicador de reserva cognitiva en la evaluación del deterioro cognitivo ligero. Anales de Psicología, 30(3), 1115-1121. doi: 10.6018/ analesps.30.3.158481

Manly, T., Owen, A. M., McAvinue, L., Datta, A., Lewis, G. H., Scott, S. K., ... Robertson, I. H. (2003). Enhancing the Sensitivity of a Sustained Attention Task to Frontal Damage: Convergent Clinical and Functional Imaging Evidence. Neurocase, 9(4), 340-349. doi: 10.1076/neur.9.4.340.15553

Marioni, R. E., Proust-Lima, C., Amieva, H., Brayne, C., Matthews, F. E., Dartigues, J. F., \& Jacqmin-Gadda, H. (2015). Social activity, cognitive decline and dementia risk: a 20-year prospective cohort study. BMC Public Health, 15(1), 1089. doi: 10.1186/s12889-015-2426-6

Mayor, J., Amador, F., \& Ramírez, I. (2008). La reserva cognitiva mejora la velocidad de procesamiento de los componentes centrales del tiempo de reacción en adultos mayores pero no en jóvenes. Revista Cubana de Salud y Trabajo, 9(1), 7-18. Recuperado de http://bvs.sld.cu/revistas/rst/

McEwen, B. S. (2007). Physiology and neurobiology of stress and adaptation: central role of the brain. Physiological Review, 87(3), 873-904. doi: 10.1152/physrev.00041.2006

Nelson, L. A., Noonan, C. J., Goldberg, J., \& Buchwald, D. S. (2013). Social Engagement and Physical and Cognitive Health Among American Indian Participants in the Health and Retirement Study. Journal of CrossCultural Gerontology, 28(4), 453-463. doi: 10.1007/ s10823-013-9213-6
Nithianantharajah, J., \& Hannan, A. J. (2009). The neurobiology of brain and cognitive reserve: mental and physical activity as modulators of brain disorders. Progress in Neurobiology, 89(4), 369-382. doi: 10.1016/j.pneurobio.2009.10.001

Pillemer, S. C., \& Holtzer, R. (2016). The differential relationships of dimensions of perceived social support with cognitive function among older adults. Aging \& Mental Health, 20(7), 727-735. doi: 10.1080/13607863.2015.1033683

Rodriguez-Laso, A., Zunzunegui, M. V., \& Otero, A. (2007). The effect of social relationships on survival in elderly residents of a Southern European community: A cohort study. BMC Geriatrics, 7(19), 1-12. doi: 10.1186/1471-2318-7-19

Rosselli, M., Jurado, M. B., \& Matute, E. (2008). Las Funciones Ejecutivas a través de la Vida. Revista Neuropsicología, Neuropsiquiatría Y Neurociencias, 8(1), 23-46. Recuperado de http://neurociencias.udea.edu.co/revista/PDF/REVNEU RO_vol8_num1_5.pdf

Saczynski, J. S. (2006). The Effect of Social Engagement on Incident Dementia: The Honolulu-Asia Aging Study. American Journal of Epidemiology, 163(5), 433-440. doi: 10.1093/aje/kwj061

Scarmeas, N., \& Stern, Y. (2003). Cognitive Reserve and Lifestyle. Journal of Clinical and Experimental Neuropsychology (Neuropsychology, Development and Cognition: Section A), 25(5), 625-633. doi: 10.1076/ jcen.25.5.625.14576

Seeman, T., Lusignolo, T., Albert, M., \& Berkman, L. (2001). Social relationships, social support, and patterns of cognitive aging in healthy, high-functioning older adults: MacArthur studies of successful aging. Health Psychology, 20(4), 243-255. doi: 10.1037//0278-6133.20.4.243

Seeman, T., Miller-Martinez, D., Stein Merkin, S., Lachman, M., Tun, P., \& Karlamangla, A. (2011). Histories of Social Engagement and Adult Cognition: Midlife in the U.S. Study. The Journals of Gerontology Series B: Psychological Sciences and Social Sciences, 66 B (S1), i141-i152. doi: 10.1093/geronb/gbq091

Segel-Karpas, D., \& Lachman, M. E. (2016). Social contact and cognitive functioning: The role of personality. The journals of gerontology. Series B, Psychological sciences and social sciences, 73(6), 974-984. doi: 10.1093/geronb/gbw079

Skakkebæk, A., Moore, P. J., Pedersen, A. D., Bojesen, A., Kristensen, M. K., Fedder, J., ... Gravholt, C. H. (2017). The role of genes, intelligence, personality, and social engagement in cognitive performance in Klinefelter syndrome. Brain and Behavior, 7(3), e00645. doi: 10.1002/brb3.645

Soto-Añari, M., Flores-Valdivia, G., \& Fernández-Guinea, S. (2013). Nivel de lectura como medida de reserva cognitiva en adultos mayores. Revista de Neurologia, 56(2), 79-85.

Stern, Y. (2009). Cognitive reserve. Neuropsychologia, 47(10), 2015-2028. doi: 10.1016/j.neuropsychologia.2009.03.004

Tun, P. A., Miller-Martinez, D., Lachman, M. E., \& Seeman, T. (2013). Social strain and executive function across the lifespan: The dark (and light) sides of social engagement. 
Neuropsychology, Development, and Cognition. Section B, Aging, Neuropsychology and Cognition, 20(3), 320-338. doi: 10.1080/13825585.2012.707173

Umberson, D., \& Karas, J. (2010). Social Relationships and Health: A Flashpoint for Health Policy. Journal of Health and Social Behavior, 51(S1), S54-S66. doi: 10.1177/0022146510383501

Wechsler, D. (2012). Escala de inteligencia de Wechsler para adultos-IV, WAIS-IV. Manual de aplicación. Mexico, D. F.: Manual Moderno.

Wilson, R. S., Boyle P. A., James, B. D., Leurgans, S. E., Buchman, A. S., \& Bennett, D. A. (2015). Negative social interactions and risk of mild cognitive impairment in old age. Neuropsychology, 29(4), 561-570. doi: 10.1037/ neu0000154

Wilson, R. S., Krueger, K. R., Arnold, S. E., Schneider, J. A., Kelly, J. F., Barnes, L. L., ... Bennett, D. A. (2007). Loneliness and Risk of Alzheimer Disease. JAMA Archives of General Psychiatry, 64(2), 234-240. doi: 10.1001/ archpsyc.64.2.234
Zainab, N., \& Naz, H. (2017). Daily living functioning, social engagement and wellness of older adults. Psychology, Community \& Health, 6(1), 93-102. doi: 10.5964/pch. v6i1.213

Zhou, Z., Wang, P., \& Fang, Y. (2018). Social Engagement and Its Change are Associated with Dementia Risk among Chinese Older Adults: A Longitudinal Study. Scientific Reports, 8(1), 1551. doi: 10.1038/s41598-017-17879-w

Zuelsdorff, M. L., Koscik, R. L., Okonkwo, O. C., Peppard, P. E., Hermann, B. P., Sager, M. A., ... Engelman, C. D. (2018). Reliability of a novel social activity questionnaire: Perceived social support and verbal interaction in the Wisconsin Registry for Alzheimer's Prevention. Journal of Aging and Health, 30(2), 305-320. doi: 10.1177/0898264316674812

Zunzunegui, M. V., Alvarado, B. E., Del Ser, T., \& Otero, A. (2003). Social networks, social integration, and social engagement determine cognitive decline in community-dwelling Spanish older adults. The Journals of Gerontology. Series B, Psychological Sciences and Social Sciences, 58(2), S93-S100. doi: 10.1093/geronb/58.2.S93 